\title{
SUBREPRESENTATIONS OF DIRECT INTEGRALS AND FINITE VOLUME HOMOGENEOUS SPACES
}

\author{
ELLIOT C. GOOTMAN
}

\begin{abstract}
We prove a result on representations of separable $C^{*}$-algebras which has application to, and was in fact motivated by, a problem concerning relations between unitary representations of a second countable locally compact group $G$ and those of a closed subgroup $K$, when $G / K$ is of finite volume. The result is that if an irreducible representation $\pi$ is contained in $\int_{X} \pi_{x} d \mu(x)$, then $\pi \subseteq \pi_{x}$ for all $x$ in a set of positive measure. With $G$ and $K$ as above, it follows that for each $\pi \in \hat{G}$ there exists $\sigma \in \hat{K}$ with $\pi \subseteq U^{\sigma}$, the induced representation. Frobenius reciprocity type results are derived as further consequences.
\end{abstract}

1. Introduction. In $[1, \S 4]$ the question is raised of whether a group representation which is equivalent to a direct sum of irreducibles can also be equivalent to some nontrivial direct integral of other irreducibles. Theorem 2.1 below provides a negative answer to this question, and can be used to weaken the hypotheses of several theorems in $[\mathbf{1}, \S 4]$. The proof is straightforward and elementary but seems to have been overlooked.

Let $G$ be a second countable locally compact group and $K$ a closed subgroup such that the quotient space $G / K$ is of finite volume. An immediate consequence of Theorem 2.1 is that for each $\pi \in \hat{G}$, the space of unitary equivalence classes of irreducible representations of $G$, there exists $\sigma \in \hat{K}$ such that $\pi$ is a subrepresentation of the induced representation $U^{\sigma}$. This result extends Theorem 2.3 of [4], where it is assumed that $G / K$ is also compact, and provides additional evidence for the conjecture, known to hold when $K$ is normal [4, Theorem 2.2], that if $K$ is any closed subgroup of $G$ such that $G$ acts amenably on $G / K$, then each $\pi \in \hat{G}$ is weakly contained in $U^{\sigma}$ for some $\sigma \in \hat{K}$. As further consequences of the above results, we obtain a necessary condition on $\pi \in \hat{G}$ in order that it satisfy Frobenius reciprocity with respect to all $\sigma \in \hat{K}$, and prove that this condition is also sufficient when $K$ is normal in $G$.

Throughout this paper, all groups are assumed to be second countable and all Hilbert spaces and algebras separable. We use the same letter $\pi$ for a unitary representation of a group $G$ and the corresponding representation of the group $C^{*}$-algebra $C^{*}(G)$, and we write $\mathcal{H}(\pi)$ for the Hilbert space of $\pi, \pi^{p}$ for the representation of a $C^{*}$-algebra determined by a positive functional $p$ on the algebra, and $\pi \subseteq \sigma$ to mean that $\pi$ is a subrepresentation of $\sigma$. The reader is referred to $[3, \S \S 3-4]$ for material on $C^{*}$-algebras associated with transformation groups.

Received by the editors September 10, 1982.

1980 Mathematics Subject Classification. Primary 22D30, 46L05.

'Supported in part by a National Science Foundation Research Grant. 


\section{Containment and Frobenius reciprocity.}

THEOREM 2.1. Let $X$ be a Borel space, $\mu$ a probability measure on $X$, and $x \rightarrow \pi_{x} a$ measurable field of representations of a separable $C^{*}$-algebra $A$. If $\pi$ is an irreducible representation of $A$ and $\pi \subseteq \int_{X} \pi_{x} d \mu(x)$, then $\pi$ is unitarily equivalent to a subrepresentation of $\pi_{x}$ for all $x$ in a measurable set $E$ of strictly positive measure.

Proof. Let $y$ be a unit vector in $\mathcal{H}(\pi)$ given by a measurable vector field $x \rightarrow y_{x}$. The set $E \equiv\left\{x \in X: y_{x} \neq 0\right\}$ is a measurable set of strictly positive measure, and as $y$ is cyclic for $\pi$ it is clear that $\pi \subseteq \int_{E} \pi_{x} d \mu(x)$. We work only with $E$ from now on and write $\mu$ for the restriction of $\mu$ to $E$. By replacing $\mu$ by an equivalent measure $\nu$ on $E$, which will be a probability measure, and whose Radon-Nikodym derivative $(d \nu / d \mu)(x)=\left\|y_{x}\right\|^{2}$, we may assume $\left\|y_{x}\right\|=1 \forall x \in E$.

For $a \in A, x \in E$, let $p(a)=\langle\pi(a) y, y\rangle, p_{x}(a)=\left\langle\pi_{x}(a) y_{x}, y_{x}\right\rangle$. The space $\mathcal{S}(A)$ of positive functionals on $A$ of norm $\leqslant 1$ is a compact convex set in the weak*-topology, and if $\delta(A)$ is given the Borel structure generated by this topology, it follows from the measurability of the functions $x \rightarrow p_{x}(a), a \in A$, that the map $\Phi$ : $E \rightarrow \delta(A)$, given by $\Phi(x)=p_{x}$, is also measurable, so that for $B$ Borel $\subseteq \delta(A)$, $\Phi^{-1}(B)$ is measurable in $E$. For such $B,\left(\Phi_{*} \nu\right)(B) \equiv \nu\left(\Phi^{-1}(B)\right)$ defines a regular probability measure on $\delta(A)$. It follows that

$$
p(a)=\int_{E} p_{x}(a) d \nu(x)=\int_{\delta(A)} q(a) d\left(\Phi_{*} \nu\right)(q), \quad a \in A
$$

Hence, by Bauer's theorem $\left[9\right.$, p. 8], $\Phi_{*} \nu$ is precisely the point mass at $p$, so $p_{x}=p$ a.e. on $E$. Thus $\pi \cong \pi^{p} \cong \pi^{p_{x}} \subseteq \pi_{x}$ a.e. on $E$, and we are done.

For a representation $\sigma$ of a closed subgroup $K$ of $G$, write $\left\langle U^{\sigma}, M^{\sigma}\right\rangle$ for the induced system of imprimitivity based on $(G, G / K)$, and $L^{\sigma}$ for the corresponding representation of the crossed product algebra $C^{*}(G \times G / K)($ see $[3, \S \S 3-4])$. By the proof of Theorem 2.1, we have

Proposition 2.2. Let $G$ be a second countable locally compact group and $K$ a closed subgroup, with homogeneous space $G / K$ of finite volume. Let $\pi \in \hat{G}$ and suppose $\left.\pi\right|_{K}$ can be decomposed as a direct integral $\int \sigma d \nu(\sigma)$. Then $\pi \subseteq U^{\sigma}$ a.e. $(d \nu)$.

Proof. It is well known that $\pi \subseteq U^{\left.\pi\right|_{K}}$ (see [8], for example), and that inducing preserves direct integrals, so that $\left.U^{\pi}\right|_{K}=\int U^{\sigma} d \nu(\sigma)$. By Theorem 2.1, we need only choose a unit vector $y$ in $\mathcal{H}(\pi) \subseteq \mathcal{H}\left(U^{\left.\pi\right|_{K}}\right)=\int \mathcal{H}\left(U^{\sigma}\right) d \nu(\sigma)$ given by a measurable vector field $\sigma \rightarrow y_{\sigma}$ such that $y_{\sigma} \neq 0$ a.e. $(d \nu)$. However, any unit vector $y$ in $\mathcal{H}(\pi)$ is cyclic for the representation $L^{\left.\pi\right|_{K}}$ of $C^{*}(G \times G / K)$, or equivalently, for the algebra of operators generated by $U^{\left.\pi\right|_{K}}$ and $M^{\left.\pi\right|_{K}}$. This can be verified routinely and we omit the details. As $L^{\left.\pi\right|_{K}}=\int L^{\sigma} d \nu(\sigma)$, it follows that $y_{\sigma} \neq 0$ a.e. $(d \nu)$.

When $G / K$ is of finite volume, representations of $G$ and of $K$ partially satisfy Frobenius reciprocity relations. Let $d(\pi, \tau)$ denote the dimension of the space of intertwining operators between two representations $\pi$ and $\tau$. Then if $\pi$ is a representation of $G$ and $\sigma$ is a representation of $K, d\left(\sigma,\left.\pi\right|_{K}\right) \leqslant d\left(\pi, U^{\sigma}\right)([8, \S 4]$ and [5, Corollary 4.8]), while a known sufficient condition that equality holds is that $\pi$ be finite dimensional $([8, \S 4]$ and $[6$, Theorem 8.2]). Leaving aside questions of multiplicity and considering only irreducible representations $\pi \in \hat{G}, \sigma \in \hat{K}$, one has the 
weaker results that (a) $\left.\sigma \subseteq \pi\right|_{K} \Rightarrow \pi \subseteq U^{\sigma}$ and (b) if $\pi$ is finite dimensional, then $\left.\pi \subseteq U^{\sigma} \Rightarrow \sigma \subseteq \pi\right|_{K}$, also. Proposition 2.2 may be viewed as stating that $\pi \subseteq U^{\sigma}$ for "almost all" $\sigma$ appearing (not necessarily as atoms) in a particular direct integral decomposition of $\left.\pi\right|_{K}$, thus providing a partial generalization of implication (a) above.

Theorem 2.1 and Proposition 2.2 are used below to obtain a necessary condition on $\pi \in \hat{G}$ for $\pi$ to satisfy implication (b) above, for all $\sigma \in \hat{K}$. This condition is weaker than finite dimensionality, and we prove it is also sufficient when $K$ is normal.

Proposition 2.3. Let $G / K$ be of finite volume, and let $\pi \in \hat{G}$. If $\pi \subseteq U^{\sigma}$ implies $\left.\sigma \subseteq \pi\right|_{K}, \forall \sigma \in \hat{K}$, then $\left.\pi\right|_{K}$ can be decomposed as a direct sum of elements of $\hat{K}$.

Proof. There exists a standard Borel space $X$ with probability measure $\nu$ and a measurable field $x \rightarrow \sigma_{x}$ of irreducible representations of $K$ such that $\left.\pi\right|_{K}=$ $\int_{X} \sigma_{x} d \nu(x)$ [2, Theorem 8.5.2]. By the hypothesis on $\pi$ and Proposition 2.2, there exists a measurable subset $E$ of $X$ with $\nu(E)=1$, such that $\left.\sigma_{y} \subseteq \pi\right|_{K}=\int_{X} \sigma_{x} d \nu(x)$, $\forall y \in E$. For each $y \in E$, let $X_{y}=\left\{x \in X: \sigma_{x} \cong \sigma_{y}\right\}$. Then $X_{y}$ is measurable, since singletons in $\hat{K}$ are Borel and the map of $X \rightarrow \hat{K}$, sending $x$ into the unitary equivalence class of $\sigma_{x}$, is Borel [7,pp. 76-81]. By Theorem 2.1 and the fact that all the $\sigma$ 's are irreducible, it follows that each $X_{y}$ is of positive measure. Since $X$ has finite measure and two sets of the form $X_{y}$ are either identical or disjoint, it is clear that there are at most countably many such sets, say $X_{i}, 1 \leqslant i \leqslant \infty$. Then $E \subseteq$ $\cup_{i=1}^{\infty} X_{i} \subseteq X$ and $\left.\pi\right|_{K}=\sum_{i=1}^{\infty} \oplus \int_{X_{i}} \sigma_{x} d \nu(x)$. As $X$ is a standard Borel space, the restriction of $\nu$ to $X_{i}$ is a standard measure and the result follows by Proposition 8.1 .7 of $[2]$.

Proposition 2.4. Let $G$ be a second countable locally compact group, let $K$ be a closed normal subgroup such that $G / K$ is compact, and let $\pi \in \hat{G}$. Then $\pi \subseteq U^{\sigma}$ implies $\left.\sigma \subseteq \pi\right|_{K}, \forall \sigma \in \hat{K}$, if and only if $\left.\pi\right|_{K}$ can be decomposed as a direct sum of elements of $\hat{K}$. This in turn occurs if and only if $\left.\pi\right|_{K}$ can be based on a finite G-orbit in $\hat{K}$.

Proof. Assume $\left.\pi\right|_{K}$ can be decomposed as a direct sum of elements of $\hat{K}$, and let $\sigma \in \hat{K},\left.\sigma \subseteq \pi\right|_{K}$. Then $\pi \subseteq U^{\sigma}$ (§4 of [8] or Corollary 4.8 of [5]), and $\left.\sigma \subseteq \pi\right|_{K} \subseteq$ $\left.U^{\sigma}\right|_{K}=\int_{G / K} x \sigma d x$ [6, Theorem 12.1], where $d x$ is Haar measure on $G / K$ and, for $x \in G, x \sigma$ is the unitary equivalence class of the representation of $K$ given by $k \rightarrow \sigma\left(x^{-1} k x\right), k \in K$. As $k \sigma \cong \sigma$ for all $k \in K$, the integral is indeed defined over $G / K$. Let $G_{\sigma} \equiv\{g \in G: g \sigma \cong \sigma\}$. A priori, $G_{\sigma}$ need not even be closed in $G$ unless $K$ is type I. By Theorem 2.1 and the irreducibility of each $x \sigma$, however, $G_{\sigma} / K$ is of positive Haar measure in $G / K$, and is thus open in $G / K$. Thus $G_{\sigma}$ is open in $G$, $G / G_{\sigma}$ is discrete, hence finite, and if $\left\{x_{1}, \ldots, x_{n}\right\}$ is a set of coset representatives for $G_{\sigma}$ in $G$,

$$
\left.\left.\pi\right|_{K} \subseteq U^{\sigma}\right|_{K}=\int_{G / K} x \sigma d x=l \cdot \sum_{i=1}^{n} \oplus x_{i} \sigma, \quad \text { where } l=\left[G_{\sigma}: K\right]
$$


As $\left.x\left(\left.\pi\right|_{K}\right) \cong \pi\right|_{K}$ for all $x$ in $G$, it follows that $\left.\pi\right|_{K}$ is indeed based on the finite $G$-orbit $\left\{x_{1} \sigma, \ldots, x_{n} \sigma\right\}$ in $\hat{K}$. Finally, if $\pi \subseteq U^{\tau}$ for some $\tau \in \hat{K}$, we have $\left.x_{i} \sigma \subseteq \pi\right|_{K}$ $\left.\subseteq U^{\tau}\right|_{K}=\int_{G / K} x \tau d x$, so as above, by Theorem 2.1, the $G$-orbit of $\sigma$ equals the $G$-orbit of $\tau$, and for some $j,\left.\tau \cong x_{j} \sigma \subseteq \pi\right|_{K}$.

\section{REFERENCES}

1. L. Baggett, $A$ unimodular Lie group having purely atomic Plancherel measure is compact, preprint.

2. J. Dixmier, Les $C^{*}$-algèbres et leurs représentations, Cahiers Scientifiques, fasc. 29, Gauthier-Villars, Paris, 1964.

3. E. G. Effros and F. Hahn, Locally compact transformation groups and $C^{*}$-algebras, Mem. Amer. Math. Soc. No. 75 (1967).

4. E. C. Gootman, Weak containment and weak Frobenius reciprocity, Proc. Amer. Math. Soc. 54 (1976), $417-422$.

5. Induced representations and finite volume homogeneous spaces, J. Funct. Anal. 24 (1977), 223-240.

6. G. W. Mackey, Induced representations of locally compact groups. I, Ann. of Math. (2) 55 (1952), 101-139.

7.. The theory of group representations, Mimeographed notes, University of Chicago, 1955.

8. C. C. Moore, On the Frobenius reciprocity theorem for locally compact groups, Pacific J. Math. 12 (1962), 359-365.

9. R. R. Phelps, Lectures on Choquet's Theorem, Van Nostrand Mathematical Studies, No. 7, Van Nostrand, Princeton, N. J., 1966.

Department of Mathematics, University of Georgia, Athens, Georgia 30602 